\title{
PHASE DIAGRAM AND SUPERCONDUCTING PROPERTIES OF AN EXACTLY SOLVABLE MODEL WITH CORRELATED HOPPING
}

\author{
F. C. Alcaraz ${ }^{1}$ and R. Z. Bariev ${ }^{1,2}$ \\ ${ }^{1}$ Departamento de Física, Universidade Federal de São Carlos, 13565-905, São Carlos, SP Brazil \\ ${ }^{2}$ The Kazan Physico-Technical Institute of the Russian Academy of Sciences, Kazan 420029, \\ Russia
}

\begin{abstract}
The model of strongly correlated electrons with the correlated hopping term and an additional interaction between holes $V$ is solved exactly in one dimension at a special point where the number of hole pairs is conserved. As a function of the interaction $V$ the phase diagram has a rich structure. There exist gapless phases with dominating density-density correlations (metallic) or pairpair correlations (superconducting) as well insulating and phase-separated phases. The stiffness constant of the conductivity, the effective transport mass, and the compressibility are also exactly calculated in several regions of the phase diagram.
\end{abstract}

PACS numbers: 75.10.Lp, 74.20-z, 71.28+d

Typeset using REVTEX 
The study of strongly correlated fermion systems has attracted considerable attention during the last decade, in particular due to the discovery of high-temperature superconductivity. Exactly solvable models, mainly those obtained using the Bethe-ansatz, have brought a very important progress in this area. Two well-studied models, which are exactly integrable in one dimension, are the Hubbard model and $t-J$ model [1-5]. In [6] a new model was proposed, which is again solvable in one dimension, and it was shown [7] to be relevant to the theory of "hole" superconductivity introduced by Hirsh [8]. According to this theory the introduction of a correlated hopping term in the Hubbard Hamiltonian leads to an attractive effective interaction between the holes and the formation of Cooper pairs. Although this picture was confirmed by a BCS-type mean-field theory it is desirable to find exact results confirming this behavior. A first step in this direction has been made in [7] where a simplified version of Hirsch's Hamiltonian has been studied in one dimension. It was found that this model has a strong tendency towards superconductivity although it does not have finite off-diagonal long-range order.

Another interesting general problem in the physics of highly correlated electronic systems is the characterization of the metallic, insulating and superconducting phases $[9,10]$. In this sense it is desirable to introduce exactly integrable models showing these phases. In this letter we introduce a model where all these phases are present. This model along with a correlated hopping term $t_{1}$ also has a static interaction $V$ between holes. We show that this model is integrable provided that $t_{1}=t$, where $t$ is the standard hopping matrix element, and study the superconducting properties at this point analytically. The phase diagram is very rich with a crossover from a region exhibiting long-range order, which corresponds to phase separation, to a regime with a complicated antiferromagnetic order, that corresponds to an insulating phase. Gapless regions with dominating superconducting pair correlation functions or dominating density-density correlations without long-range order also exist.

The Hamiltonian of the correlated hopping model with an additional interaction beetween holes on a one-dimensional lattice with $L$ sites and periodic boundary conditions is given by 


$$
\begin{aligned}
H\left(t_{1}, V\right)= & -\sum_{j=1}^{L} \sum_{\sigma= \pm 1}\left[\left(c_{j, \sigma}^{+} c_{j+1, \sigma}+c_{j+1, \sigma}^{+} c_{j, \sigma}\right)\right. \\
& \times\left(1-t_{1} n_{j+(1+\sigma) / 2,-\sigma}\right) \\
& \left.+V\left(2-n_{j, \sigma}-n_{j+1, \sigma}\right)\left(1-n_{j,-\sigma}\right)\right]
\end{aligned}
$$

where $c_{j, \sigma}$ is the annihilation operator for an electron with spin $\sigma$ at site $j$ and $n_{j, \sigma}=c_{j, \sigma}^{+} c_{j, \sigma}$. Here we choose the hopping matrix element $t=1$. The integrable correlated hopping chain [7] is a special case of (1) with $V=0$.

Firstly we will be interested in the special case $H(V)=H\left(t_{1}=1, V\right)$. Note that the analogous construction have been considered for the Hubbard model in [10-12]. The results of this consideration are quite different from ours.

In the Hamiltonian $H(V)$ only one type of processes is allowed : a hole at the site $(j, \sigma)$ may hop to an occupied neighbour site $(j \pm 1, \sigma)$ provided that the site $(j+(\sigma \pm 1) / 2,-\sigma)$ is empty. This process corresponds to the motion of the hole pair on the half of the lattice distance. In the Hamiltonian $H(V)$ there is also the static interaction between holes which corresponds to an extremely anisotropic spin-spin interaction [12]. We consider hereafter the situation where we have only pair of holes [13]. In this case we consider hole pairs as "particles" and treat this problem within the framework of the Bethe-ansatz method.

The discrete Bethe-ansatz equations are derived following the standard procedure by imposing periodic boundary conditions. Each state of the Hamiltonian is specifed by the set of charge rapidities $p_{j}$ representing the momenta of the hole pairs. All rapidities within a given set have to be different corresponding to Fermi statistics. These rapidities are determined by the Bethe-ansatz equations

$$
\begin{array}{r}
e^{2 i L p_{j}}=(-1)^{m-1} \prod_{l=1}^{m} \exp \left[i\left(p_{j}-p_{l}\right)-i \Theta\left(p_{j}, p_{l}\right)\right] \\
\exp \left[-i \Theta\left(p_{j}, p_{l}\right)\right]=S\left(p_{j}, p_{l}\right) / S\left(p_{l}, p_{j}\right) \\
S\left(p_{j}, p_{l}\right)=\left(1-2 V e^{i p_{j}}+e^{i p_{j}+i p_{l}}\right) .
\end{array}
$$

The energy and momentum of the corresponding state are given by 


$$
E=-2 \sum_{j=1}^{m}\left(\cos p_{j}+V-\mu\right)-2 L \mu, \quad P=\sum_{j=1}^{m} p_{j} ;
$$

where the chemical potential $\mu$ has been added to control the particle number.

The above equations are valid for density of holes $\rho=m / L \leq 2 / 3$. For $\rho>2 / 3$ we can choose as the reference state the state with unoccupied sites and treat again this problem by the Bethe-ansatz method. Each state of the Hamiltonian is now specified by the set of charge rapidities $k_{j}$ representing the momenta of the electrons. These rapidities are determined by the following Bethe-ansatz equations

$$
e^{i L k_{j}}=-\prod_{l=1}^{n} \exp \left[\frac{i}{2}\left(k_{l}-k_{j}\right)-i \Theta\left(k_{j}, k_{l}\right)\right]
$$

The corresponding energy and momentum are given by

$$
E=-4 L V+\sum_{j=1}^{n}\left(4 V-\mu-2 \cos k_{j}\right), \quad P=\sum_{j=1}^{n} k_{j} .
$$

We may consider $(2,3)$ and $(4,5)$ in the straighforward method [14]. In the thermodynamic limit (2) and (4) are replaced by the integral equations for the distribution functions

$$
\begin{gathered}
2 \pi R(U)=K_{1}(U)\left(1-\frac{\rho}{2}\right)-\int_{-U_{0}}^{U_{0}} K_{2}\left(U-U^{\prime}\right) R\left(U^{\prime}\right) d U^{\prime}, \\
\int_{-U_{0}}^{U_{0}} R(U) d U= \begin{cases}\frac{1}{2} \rho, & \rho \leq 2 / 3, \\
(1-\rho), & 2 / 3 \leq \rho \leq 1,\end{cases}
\end{gathered}
$$

where for $-1<V=-\cos \gamma<1$

$$
K_{\alpha}(U)=\sin (\alpha \gamma)[\cosh U-\cos (\alpha \gamma)]^{-1},(\alpha=1,2)
$$

and for $V=-\cosh \lambda<-1$

$$
K_{\alpha}(U)=\sinh (\alpha \lambda)[\cosh U-\cos (\alpha \lambda)]^{-1},(\alpha=1,2)
$$

The energy is given by

$$
\frac{1}{2 L} E=\int_{-U_{0}}^{U_{0}} \epsilon(U) d U
$$


where the dressed energy $\epsilon(U)$ is the solution of the integral equation

$$
\epsilon(U)=\epsilon_{0}(U)-\frac{1}{2 \pi} \int_{-U_{0}}^{U_{0}} K_{2}\left(U-U^{\prime}\right) \epsilon\left(U^{\prime}\right) d U^{\prime}
$$

Here for $V<1$

$$
\epsilon_{0}(U)=-2 \sin \gamma K_{1}(U)+\frac{1}{2}(1 \pm 3)(\mu+2 \cos \gamma)
$$

with sign $+(-)$ for $\rho<2 / 3(\rho>2 / 3)$, respectively. For $V<-1$

$$
\epsilon_{0}(U)=-2 \sinh \lambda K_{1}(U)+2(\mu+2 \cosh \lambda) .
$$

In all cases $\epsilon\left( \pm U_{0}\right)=0$.

At $V<-1$ and $\rho>2 / 3$, there is a gap in the energy spectrum and it corresponds to the existence of long-range order in the system. In this case the equations $(6,8)$ are not valid.

At $V>1$ there is a gap for the arbitrary concentration of holes and this state has long-range order which corresponds to phase separation at $V=-1$.

For $-1<V<1$ there is no gap in the eigenspectrum and in order to understand the superconducting properties of the model we shall investigate the long-distance behavior of the correlation functions. For this purpose we shall use two-dimensional conformal field theory [15] and analytic methods $[16,17]$ to extract finite-size corrections from the Betheansatz equations. The results of these calculations are the following.

The long-distance power-law behavior of the density-density correlation functions is given by

$$
\langle\rho(r) \rho(0)\rangle \simeq \rho^{2}+A_{1} r^{-2}+A_{2} r^{-\alpha} \cos \left(2 k_{F} r\right)
$$

where

$$
2 k_{F}=\pi \rho, \quad \rho(r)=\sum_{\sigma} c_{r \sigma}^{+} c_{r \sigma}
$$

The critical exponent $\alpha$ is expressed in terms of the dressed charge $\xi\left(U_{0}\right)$ which satisfies the integral equation 


$$
\xi(U)+\frac{1}{2 \pi} \int_{-U_{0}}^{U_{0}} K_{2}\left(U-U^{\prime}\right) \xi\left(U^{\prime}\right) d U^{\prime}=1-\frac{1}{2} \rho
$$

The Eq. (10) and (14) add to the integral equations (6) and (8) [18]. The superconducting properties of the system manifest themselves in the behavior of the pair correlation function

$$
\begin{aligned}
G_{p}(r) & =\left\langle c_{r \uparrow}^{+} c_{r, \downarrow}^{+} c_{0 \downarrow} c_{0 \uparrow}\right\rangle \simeq B r^{-\beta}, \\
\beta & =\alpha^{-1}=\frac{1}{2\left[\xi\left(U_{0}\right)\right]^{2}} .
\end{aligned}
$$

The exponent $\beta$ is plotted in Fig.1 for some values of the parameter $V$. These results are obtained by solving numerically the integral equations (6) and (10). We see from this figure that for all values of $V$ there exists a critical density $\rho_{c}$ separating a regime with dominant density-density correlations $\beta>1$ from a regime with dominant pair superconducting correlations $\beta<1$. These two regimes are represented in the phase diagram of Fig. 2 by the phases $C$ and $B$, respectively. The critical curve $\rho_{c}(V)$ is obtained numerically and is represented by the broken line. In phase $B$, where the superconducting correlation function decays slower than the density-density correlation function we have the closest analogy with a true superconducting phase. Analogous behavior of correlation functions are observed in other models $[7,19]$, but differently from these models, in the present case we have the nice feature that for increasing values of $\rho$, the exponent $\beta$ tends toward zero. This means that the system try to have a true off-diagonal long-range-superconducting order (ODLRSO). However at the same time when $\beta \rightarrow 0$, which happens for $V \rightarrow 1^{-}$, the hole compressibility, as we shall see, tends to infinity and phase separation takes place. Then at $V=1$ we have two phase transitions happening simultaneously. We may expect that in two dimensions these phase transitions happen at different places [20].

In region $D(V<-1, \rho>2 / 3)$ we have no bound pairs but a long-range "antiferromagnetic order", where hole pairs and electrons are located alternately. This is an insulating gapped phase. In order to see this we calculated the electrical conductivity in our model. This can be done by studying the Hamiltonian (1) with twisted boundary condition, where the twisting angle $\phi$ corresponds to an enclosed magnetic flux in the ring [9]. The conductiv- 
ity is directly proportional to the charge stifness $D_{c}$, which can be obtained from the change $\Delta E_{0}=D_{c} \phi^{2} / L$ of the ground-state energy $E_{0}$, for small $\phi$ and large $L$. From the charge stiffness we can define an effective transport mass by the relation

$$
m / m_{e}=D_{c}^{0} / D_{c}
$$

where $D_{c}^{0}=2 \sin (\pi \rho) / \pi$ is the charge stiffness of the non-interacting system and $m_{e}$ is the electron mass. Extending the Bethe ansatz equations (2-5) for the case of twisted boundary conditions [21] $c_{L+1, \sigma}^{ \pm}=\exp \left( \pm i \phi_{\sigma}\right) c_{1, \sigma}^{ \pm}$with $\phi_{+}=\phi_{-}=\phi$ and exploring the finite size corrections obtained from conformal invariance [15] we obtain, after some calculation

$$
D_{c}=2 v_{F} \xi^{2} / \pi, v_{F}=\epsilon^{\prime}\left(U_{0}\right) /\left(4 \pi R\left(U_{0}\right)\right)
$$

where by prime we mean derivative. In Fig. 3 we show $m / m_{e}$ obtained numerically for several values of $V$. We clearly see in this figure that for $V<-1$ as $\rho \rightarrow 2 / 3$ the transport mass diverges, which indicates that phase $D$ in Fig. 2 is an insulating phase, and at $\rho=2 / 3$ we have a Mott transition. The anomalous behavior in Fig. 1 and 3 around $\rho=2 / 3$ at $V=-0.98$ is due to the strong competition between the repulsive and kinetic energy, which for larger values of $V$ will lead to the Mott transition. This figure also shows that for $-1<V<1$ the carrier mass is around $2 m_{e}$ for moderate densities, which means that the conductivity is ruled by the motion of pairs.

For $V>1$ (phase $A$ in Fig. 2) we have bound pairs, for any density and there is a long-range order in the system. This is a region where phase separation takes place. The hole pairs are separated from the particles. In order to see this we calculated the hole compressibility $\kappa=1 /\left(\rho^{2} \frac{d^{2} E_{\rho}}{d \rho^{2}}\right)$, where $E_{\rho}$ is the ground-state energy for a given concentration $\rho$. Exploring, as before, the finite-size corrections obtained from conformal invariance [15] we obtain $\kappa=D_{c} / 2 v_{F}^{2} \rho^{2}$. In Fig. 4 we show the curves of $\kappa$ for certain values of $V$. We clearly see in this figure that $\kappa$ increases drastically for all densities as we tend toward $V=1$, indicating a phase separation for $V>1$ (phase $A$ in Fig. 2) [22].

Thus we have investigated a correlated hopping model [7] with an additional hole-hole interaction $V$ at the special point $t_{1}=1$ where the number of hole pairs is conserved and 
we have only hole pairs. We have shown that our model exhibits phase separation and Mott transition at $V=1$ and $V=-1$, respectively. At $-1<V<1$ there are regions with dominating superconducting correlations. Although the system in this region has no ODLRSO it is very close to the true superconducting state since the critical exponent of the superconducting correlation function $\beta$ tends to zero.

In the general case at $0<t_{1}<1$ desintegration of hole pairs is possible, and the number of hole pairs is not conserved. However these states are not favorable in energy and their contribution is negligible since they are separated from those considered above by a large energy gap, at least for a small density of holes. As a result, we may expect that at arbitrary $t_{1}$ the qualitative behavior of the system will be same as that considered at $t_{1}=1$. Certainly it is desirable to investigate this problem using perturbation theory and exact diagonalization of small systems.

This work was supported in part by Conselho Nacional de Desenvolvimento Científico e Tecnológico - CNPq - Brazil, and by Fundação de Amparo à Pesquisa do Estado de São Paulo - FAPESP - Brazil. 


\section{REFERENCES}

1. E. H. Lieb and F. Y. Wu, Phys. Rev. Lett. 20, 1445 (1968).

2. B. Sutherland, Phys. Rev. B 123795 (1975).

3. P. Schlottmann, Phys. Rev. B 36, 5177 (1987).

4. P. A. Bares and G. Blatter, Phys. Rev. Lett. 64, 2567 (1990).

5. V. E. Korepin and F. H. L. Essler , Exactly Solvable Models of Strongly Correlated Electrons (World Scientific, Singapore, 1994).

6. R.Z.Bariev, J. Phys. A:Math.Gen. 24, L549 (1991).

7. R. Z. Bariev, A. Klümper, A. Schadschneider and J. Zittartz, J. Phys. A:Math.Gen. 26, 1249; 4863 (1993).

8. J. E. Hirsch, Phys. Lett. A 134, 451 (1989); Physica C 158326 (1989); Phys. Rev. B 43, 11400 (1991).

9. B. S. Shastry and B. Sutherland, Phys. Rev. Lett. 65, 243 (1990); D. J. Scalapino, S. R. White, and S. C. Zhang, Phys. Rev. Lett. 68, 2830 (1992); N. Kawakami and S. -K. Yang, Phys. Rev. B 44, 7844 (1991).

10. L. Arrachea and A. A. Aligia, Phys. Rev. Lett. 73, 2240 (1994); L. Arrachea, A. A. Aligia and E. Gagliano, Phys. Rev. Lett. 76, 4396 (1996).

11. F. H. L. Essler, V. E. Korepin and K. Schoutens, Phys.Rev.Lett. 68, 2960 (1992); 70, 73 (1993).

12. J.de Boer, V.E.Korepin, and A. Schadschneider, Phys. Rev. Lett. 74, 789 (1995); A. Schadschneider, Phys. Rev. 51, 10386 (1995).

13. The number of hole pairs and isolated holes are conserved separately. The general case with arbitrary number of hole pairs and isolated holes will be considered elsewhere.

14. R. J. Baxter, Exactly solved models in statistical mechanics (Academic Press, New York, 1982). 
15. J. L. Cardy, Nucl. Phys. B 270 [FS16], 186 (1986).

16. F. Woynarovich, H. P. Eckle and T. T. Truong, J. Phys. A:Math. Gen. 22, 4027 (1989).

17. N. M. Bogoliubov and V. E. Korepin, Int. J. Mod. Phys. B 3, 427 (1989).

18. Comparing Eqs. $(6,10)$ with the corresponding results for the XXZ model (see Ref. 16) we obtain simple relations between the critical exponents of these models.

19. R. Z. Bariev, Phys. Rev. B 49, 1447 (1994); R. Z. Bariev, A. Klümper, A. Schadschneider and J. Zittartz, Phys.Rev. B 50, 9676 (1994); R. Z. Bariev, A.Klümper and J.Zittartz, Europhys. Lett. 32, 85 (1995).

20. E. Dagotto, J. Riera, Y. C. Chen, A. Moreo, A. Nazarenko, F. C. Alcaraz, and F. Ortolani, Phys. Rev. B 49, 3548 (1994).

21. F. C. Alcaraz, M. N. Barber and M. T. Batchelor, Phys. Rev. Lett. 58, 771 (1987); Ann. Phys. 182, 280 (1988).

22. The large values of $\kappa$ for $V<-1$ and $\rho=2 / 3$ arise because the insulating phase $D$ has a gap. 


\section{FIGURES}

FIG. 1. The exponent $\beta$ of the pair correlation function as a function of hole density $\rho$ for some values of the interaction $V$.

FIG. 2. Phase diagram as a function of the interaction $v$ and hole density $\rho$. Phases $A$ and $D$ are gapped and correspond to phase separations and insulating phases, respectively. Phases $B$ and $C$ are gapless and corresponds to the region with dominating behavior of superconducting correlations and density-density correlations, respectively.

FIG. 3. The effective transport mass as a function of density of hole $\rho$ for some values of $V$.

FIG. 4. The hole compressibility as a function of density of hole $\rho$ for some values of $V$. 